\title{
Computational fluid dynamic modelling and simulation evaluation of the plume evacuation device efficiencies
}

\author{
F. Farshad ${ }^{1}$, H. Rieke ${ }^{1}$, L. C. LaHaye ${ }^{2}$ \& S. C. Nulu ${ }^{1}$ \\ ${ }^{1}$ University of Louisiana at Lafayette, USA \\ ${ }^{2}$ Vision Pro LLC, USA
}

\begin{abstract}
The purpose of our work has been to evaluate the fluid flow dynamics of distal and proximal handheld plume evacuation devices used during LASIK eye surgery using Computational Fluid Dynamic (CFD) Modelling.

Fluid flow dynamics studies using CFD simulations were conducted on a proximal plume evacuator, LAHayeSIK ${ }^{\mathrm{TM}}$ surgical device, and on the VISX Star $\mathrm{S} 3$, which is a distal large volume plume evacuation device. The resulting data was compared and analyzed with experimental data.

CFD results show that the proximal plume evacuation system generated a uniform laminar airflow velocity of $0.94 \mathrm{~m} / \mathrm{s}$ across the corneal surface as compared to $1.3 \mathrm{~m} / \mathrm{s}$ reported by the distal evacuation system. Flow profiles indicate high shear regions resulting in vortex formations, for the large volume distal evacuator.

The CFD simulations conducted to determine the airflow profiles generated by the two surgical plume evacuation devices concur with data obtained from experiments. Flow patterns simulated by the CFD modeling, indicate that the proximal plume evacuation devices generate a gentle laminar airflow profiles over the stromal surface. On the other hand, the distal large volume plume evacuators generate multiple regions of varying air flow velocities contributing to ineffective plume capture.
\end{abstract}

Keywords: computational fluid dynamics, fluent, LAHayeSIKTM, LASIK, plume evacuation, CFD simulation. 


\section{Introduction}

One of the ultimate goals in performing any surgical procedure is to minimize less than desirable outcomes arising from both infectious and noninfectious contaminants entering the surgical field (LaHaye et al., [1, 2]). Although not normally thought of as such, plume smoke is a by-product contaminate of excimer laser surgery. The complexities of plume formation and its rapid dynamic movements both vertically and laterally impose problems that present undesirable outcomes and present health related issues for the surgeon, patient, and nursing staff. In excimer refractive surgery a laser's accuracy, effectiveness, and reproducibility can be directly affected by how well the surgical operating environment is managed. The authors emphasize that managing the microclimate of the stromal bed during the excimer refractive procedures is the only important avenue where substantial improvements can be made through advancements in design for better outcomes and fewer health risks. Air flow dynamics generated by plume evacuation systems can have direct and indirect influences on refractive outcome. A direct effect of LASIK plume smoke is the masking effect created as the plume hangs just over the ablating stromal bed, blocking subsequent excimer pulses which can cause a measurable difference in the resulting ablation (Duffey, [3]). Researchers contend that the plume particles falling back onto the on the ablating stromal bed creates additional beam masking (Noack et al., [4]) and may be a contributing factor to the "Sands of the Sahara" syndrome (Dell, [5]).

Research has demonstrated that plume vapor condensation with precipitation contributes to visible fluid accumulation on the surface of the stromal bed during ablation. This additional regional accumulation of fluid can interfere with beam etching to cause a decrease in transmission of energy to the stroma through increased reflection and absorption of incident laser energy. This resulted in undesirable ablation, such as central islands, "hot and cold" spots, and under corrections (Oshika et al., [7]). Most corporate and physician-based nomograms are based on a certain portion of the excimer beam being blocked by plume particles (Maguen and Machat, [8], Duffey, [3]). Laser manufacturers add pulses to the nomograms based on outcome averages to "compensate" for plume masking attributes of their systems. The "blanket" method of re-mediating the numerous problems associated with plume appears to have little logic. The many potential problems associated with plume should direct one to solving the underlying issue by removing the cause and therefore the effect as opposed to simply adding additional laser pulses in an attempt to compensate.

Since the adoption of excimer refractive procedures some 15 years ago, the industry has several alternatives for plume management. The options include; (1) no plume management; (2) operating room ventilation fan generated room air blown across the surgical field; (3) laser integrated distal plume evacuation; (4) laser integrated devices which combine blown air and distal plume evacuation; and (5) handheld proximal plume evacuation devices. Because of the potential health hazards associated with plume, we have chosen to compare systems that 
are, in theory, designed to only remove plume. Experimental analysis and Computational Fluid Dynamics were used to comprehend the dynamics of the plume generated during the LASIK surgery. Two types of plume evacuation devices, the proximal plume evacuation device - LAHayeSIK ${ }^{\text {TM }}$ Surgical System and the distal plume evacuation system - VISX Star S3 are compared for their effectiveness in design to remove plume generated during LASIK surgery. Our CFD results of this study emphasize modern computational techniques like CFD, which can be used with a great effect in determining the best design technique for medical equipment design.

\section{CFD simulation of the proximal LAHayeSIK ${ }^{\mathrm{TM}}$ plume evacuation system}

The flow domain for the proximal plume evacuation system is identified as the path inside and outside the handpiece in the vicinity of the stromal surface, where the plume particles travel under the influence of the plume evacuation force. Figure 1 shows the plume flow domain of the proximal plume evacuation system that is to be modeled.

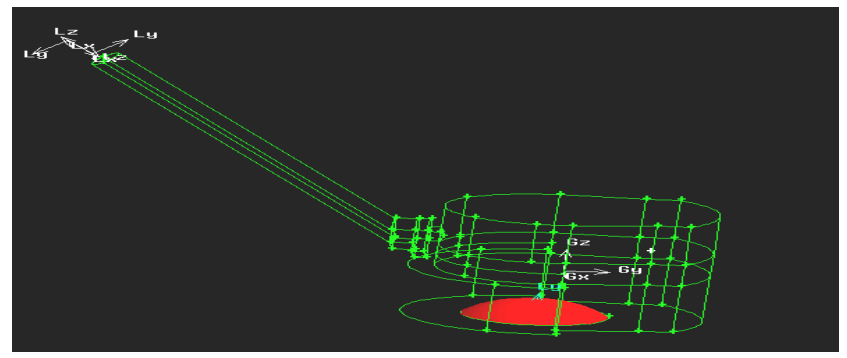

Figure 1: $\quad$ Solid model (domain) of the LAHayeSIK ${ }^{\mathrm{TM}}$ surgical device.

The solid model of the domain is created in FLUENT's GAMBIT using the basic geometrical tools such as edges, faces, and volumes. The design of the solid model incorporates all the nuances in the model and the exact measurements of angles and distances. The model is then meshed using various meshing strategies to come up with the best quality mesh. The mesh can be used by FLUENT to solve the numerical equations without any divergence problems. Figure 2 shows the meshed model of the plume evacuation function of the LAHayeSIK $^{\mathrm{TM}}$ surgical device. The meshed model from GAMBIT is then set up inside the FLUENT console after performing a grid check for negative volumes and inconsistent meshing. Thus, the solution is setup with the required parameters and boundary conditions and is iterated for convergence with a constant monitoring of the solution using the residuals. The convergence criteria are set to be $10^{-6}$ and once the residuals reach this prescribed value, the solution is said to have converged and the data is post processed and the results analyzed. 


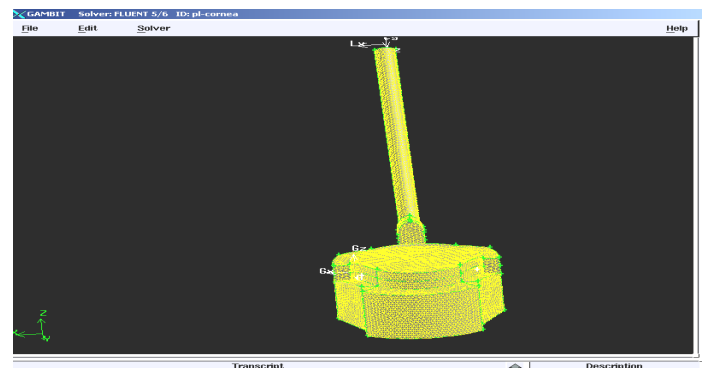

Figure 2: Meshed model of the plume evacuation flow domain of the LAHayeSIK $^{\mathrm{TM}}$ surgical device.

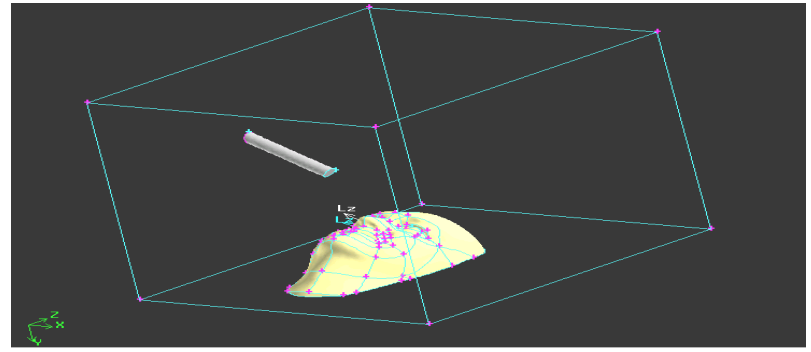

Figure 3: Solid model of the plume evacuation flow domain of the distal plume evacuation surgical device.

\section{CFD simulation of the distal VISX Star S3 plume evacuation system}

The flow domain for the distal plume evacuation system is identified as the path in the vicinity of the stromal surface from the evacuation tube, where the plume particles travel under the influence of the suction force. Figure 3 presents the plume flow domain that has been modeled. To better capture the effects of facial features such as the nose, setting of the eyes, and the evacuation tube's influence on the flow of room air. A modeled face is in the surgical position as the subject that under goes surgery. Notice the considerably sharp features of the nose and reasonably deep-set eyes in figure 3. The model is then meshed using various meshing strategies to come up with the best quality mesh, which can be used by FLUENT to solve the numerical equations without any divergence problems. The plume evacuation function of the VISX surgical device was constructed as a mesh model. The meshed model from GAMBIT is then exported to the solver, which is FLUENT in the present CFD analysis.

\section{CFD simulation results}

The CFD results obtained in this simulation indicate some important flow aspects of the plume under the evacuation field generated by the plume 
evacuation devices. The proximal plume evacuation system shows a more effective evacuation influence on the microclimate over the corneal surface and thus assists in the onsite plume removal without giving the plume particles a longer time of travel. It is noted that the longer the plume particles are in the vicinity of the corneal surface, the more chances for plume masking and other complications. The proximal plume evacuation technique allows a quick $360^{\circ}$ on site plume removal technique along the circumference of the cornea with high velocity gradients created just above the corneal surface. The design of the ports is such that low and uniform velocity fields are generated over the corneal surface itself, thereby reducing the risk of over dehydrating the cornea.

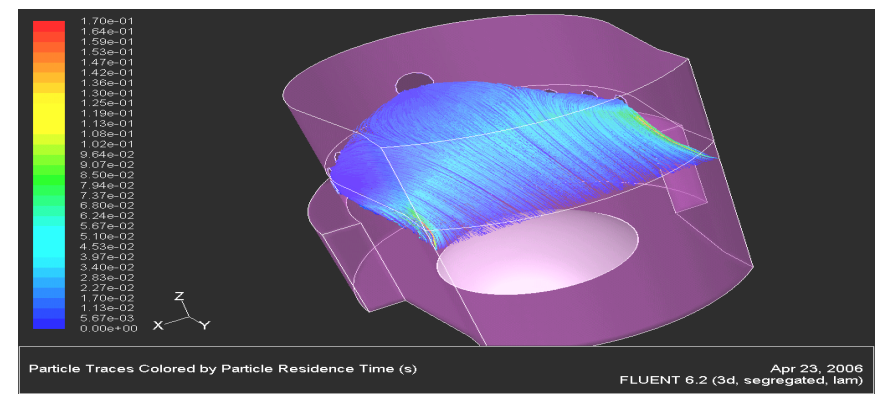

Figure 4: $\quad$ Flow path of the plume particles which are situated at height of the plume channel of the LAHayeSIK ${ }^{\mathrm{TM}}$ surgical device. Simulation results show that all the plume is captured by the seven strategically placed ports.

Figure 4 shows the trajectories of the plume particles that are generated under the influence of the evacuation force of the LAHayeSIK ${ }^{\mathrm{TM}}$ plume evacuation function. The particles are created at a distance away from the corneal surface and the seven ports at a height of $2 \mathrm{~cm}$ from the corneal surface. The simulation shows that the entire plume is being captured by the seven strategically placed ports on the circumference of the handpiece.

Simulating the particle paths to evaluate the velocity functions with a certain direction over the surface is a common practice to either create a set of points and plot their velocities or simulate the paths of the particle trajectories. In FLUENT, rakes are used to serve the same purpose. Rakes are a predetermined number of points between two specified endpoints.

Figures 5 and 6 simulate the actual flow path of the plume particles that are generated during the actual surgery. These simulations show that not only the entire plume is effectively captured by the seven ports but also the flow paths indicate that there are no vortices or turbulent behavior during the plume travel.

Contour maps show the velocity profiles over specified cross sections of a fluid domain (Figs. 7 and 8). These maps play an important role in indicating the change in velocities along the radial direction of the device. Figure 7 presents a contour map of simulated velocities generated by the plume evacuation function of the LAHayeSIK ${ }^{\mathrm{TM}}$ surgical device at the corneal height. It shows low 
velocities with uniform profiles, which is important to minimize uneven dehydration of the cornea. Figure 8 shows the contour map of simulated velocities generated by the plume evacuation function of the LAHayeSIK ${ }^{\mathrm{TM}}$ surgical device at the plume channel height, which is about $3 \mathrm{~cm}$ from the cornea. This indicates that there is a high velocity field generated centimeters above the corneal surface that assists in the complete removal of the plume particles from the microclimate with no escape into the operating room, as shown by the particle paths. Figure 9 shows the plot of velocities on the rakes. This plot is an indication of the directional velocity changes as one goes away from the plume evacuation ports. The flatness of the curves indicates that there are minimal changes in the velocities in the radial direction and that an overall uniform evacuation is achieved by the combined effect of the seven evacuation ports.

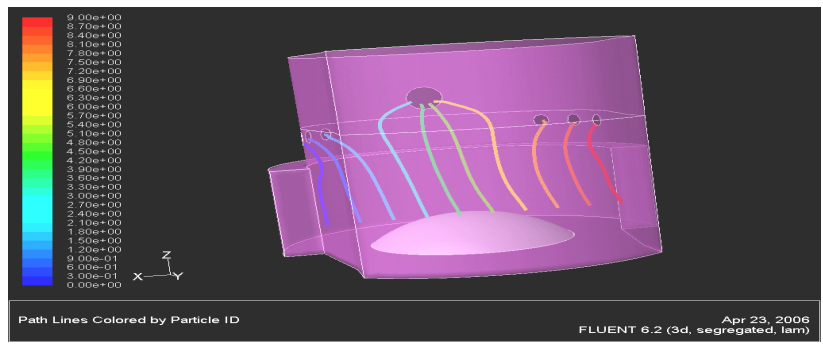

Figure 5: $\quad$ Flow path of the plume particles situated at different places on the cornea under the influence of the LAHayeSIK ${ }^{\mathrm{TM}}$ plume evacuation function.

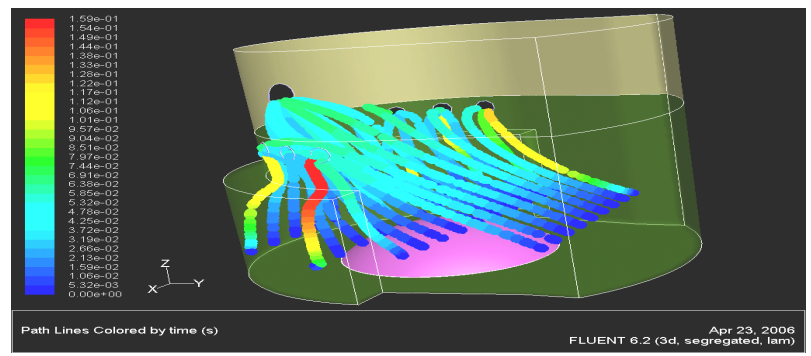

Figure 6: Flow path of the plume particles situated at different places on the cornea under the influence of the LAHayeSIK ${ }^{\mathrm{TM}}$ plume evacuation function. A complete capture is shown of all the plume particles generated at the corneal surface.

CFD simulations used the distal large volume VISX Star S3 plume evacuator to compare the CFD results with the experimental results and to delve deeper into the flow dynamics of distal plume evacuators. Figure 10 shows the velocity vectors generated over the facial contours by the VISX Star S3 plume evacuation 
function achieved by employing the $22 \mathrm{~mm}$ diameter tube. The vector field indicates uneven velocity gradients with high and low velocity fields in the vertical plane, which create shear and cause vortices resulting in a high degree of turbulence. These vectors support the high turbulence and vortices observed during experiments with the artificial plume (Figure 11).

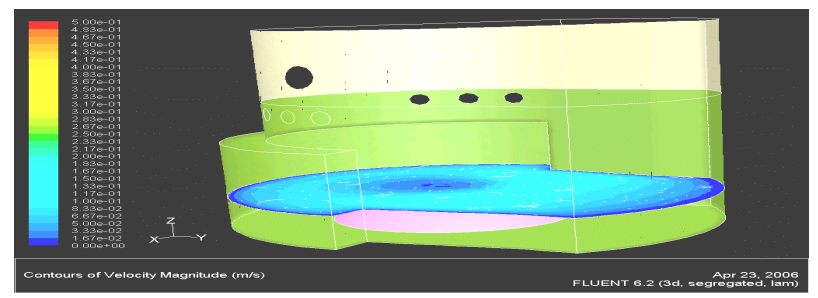

Figure 7: Contour map of velocities generated by the plume evacuation function of the LAHayeSIK ${ }^{\mathrm{TM}}$ surgical device at the corneal height.

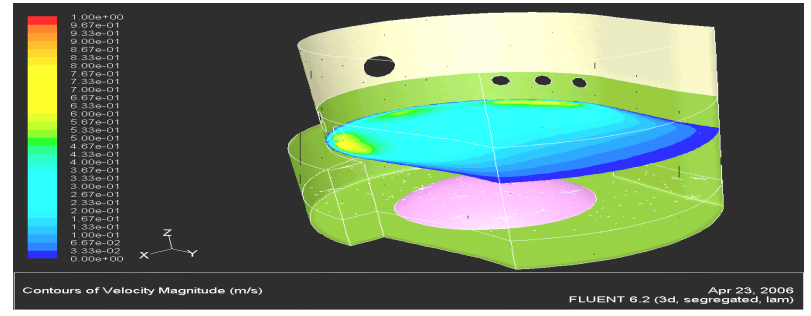

Figure 8: Contour map of velocities generated by the plume evacuation function of the LAHayeSIK ${ }^{\mathrm{TM}}$ surgical device at the plume channel height which is about $3 \mathrm{~cm}$ from the cornea.

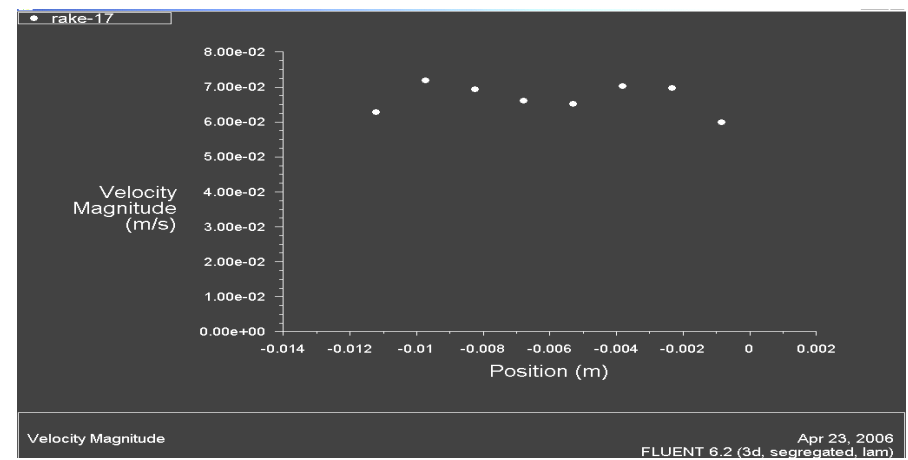

Figure 9: Plot of velocities with respect to position generated over the cornea in the direction of a rake. 


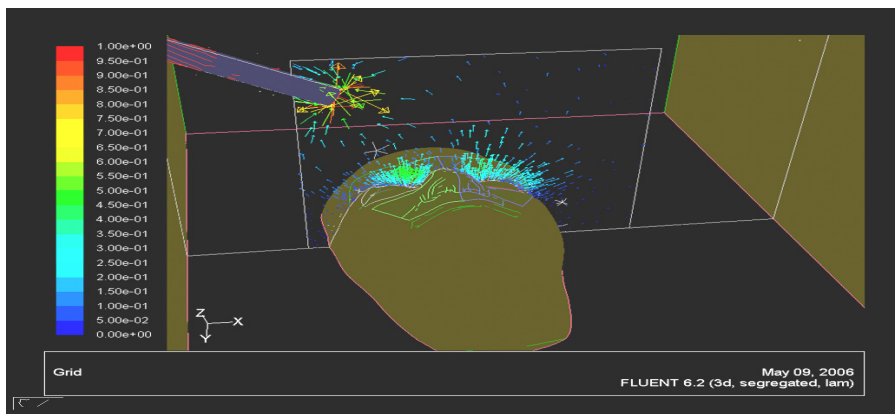

Figure 10: Vector plot of the velocities generated by the VISX Star S3 distal plume evacuation device. The vertical plane of interest where the experimental velocities are measured.

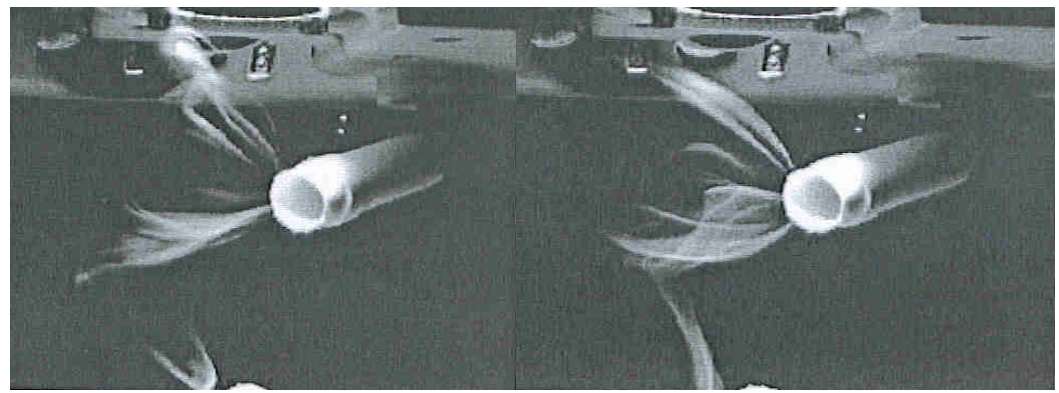

Figure 11: Photographs of the formation of vortices and plume escape in the field of a large volume distal plume evacuator documenting inefficient plume capture during LASIK surgery.

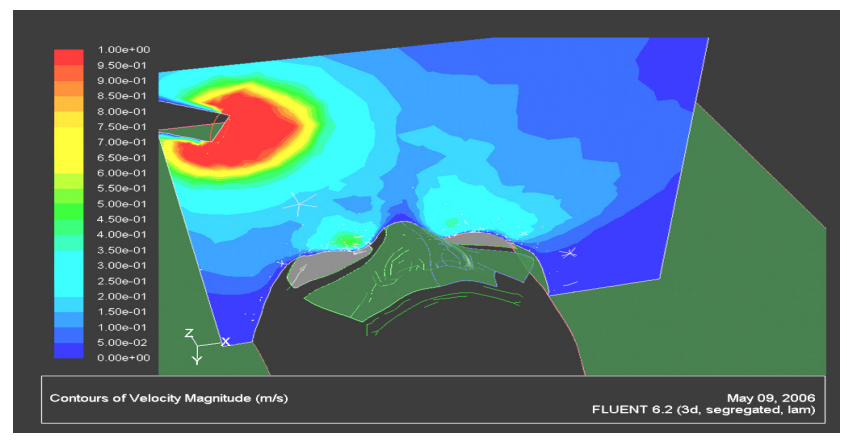

Figure 12: Contour map of the velocity field generated by VISX Star S3 large volume plume evacuator in a plane perpendicular to corneal surface.

Figure 12 is the contour map of the velocities on the plane perpendicular to the corneal surface. These contour maps show similar profiles as obtained by 
experimental studies, thereby validating the CFD simulation results. Figure 13 shows the trajectories of the plume particle paths under the influence of the distal plume evacuation velocity field generated by the distal large volume VISX Star S3 plume evacuator. The high shear generated by the high and low velocity fields in close proximity of the corneal surface cause turbulence and vortices. The non-uniform flow patterns cause escape of plume as shown in Figure 13.

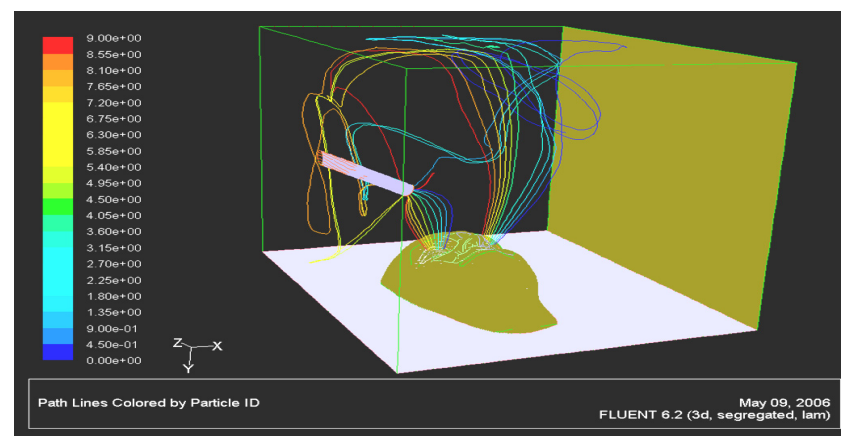

Figure 13: Path lines of trajectories of plume particles in the influence of the plume evacuation force field of the VISX Star S3 device.

\section{Conclusions}

Both the experimental and CFD simulation results indicate that the proximal plume evacuation systems are designed to better handle the plume evacuation as compared to the distal large volume plume evacuators. The proximal plume evacuators, owing to their proximity of the plume evacuation ports from the stromal surface have a greater effect in removing the generated plume. On the other hand, the particle trajectory simulations of the large volume evacuators indicate escape of particles and an inefficient plume removal. Also the presence of varying velocity fields just above the stromal surface results in high shear and thus there is a good possibility of vortex formations due to turbulence. This study concludes that the proximal plume evacuation systems such as LAHayeSIK ${ }^{\mathrm{TM}}$ are better designed to remove the plume generated during LASIK surgery as opposed to existing large volume evacuation technology.

\section{References}

[1] LaHaye, L.C., Rieke, H.H., and Farshad, F.F., Is Cleaner EXCIMER REFRACTIVE PROCEDURES Possible? Part I, Ophthalmology Management, Oct 2005, pp. 97-99.

[2] LaHaye, L.C., Rieke, H.H., and Farshad, F.F., Is Cleaner EXCIMER REFRACTIVE PROCEDURES Possible? Part 2, Ophthalmology Management, Jan 2006, pp. 45-46. 
136 Computational Methods and Experimental Measurements XIII

[3] Duffey, R., Central Islands and Decentered Ablations After Excimer Refractive Procedures, International Ophthalmology Clinics 2000, 40, pp. 93-101.

[4] Noack et al., Influence of Ablation Plume Dynamics on the Formation of Islands in Excimer Laser Photorefractive Keratectomy, Ophthalmology 1997, 104(5), pp. 823-830.

[5] Dell, New System permits safe, more effective plume evacuation, Ophthalmology Times, April 2003.

[6] Charle, K., Effects of Laser Plume Evacuation on Laser in situ Keratomileusis Outcomes, Journal of Refractive Surgery, June 2002 (Suppl), pp. 340-341.

[7] Oshika et al., Corneal hydration and central islands after excimer laser photorefractive keratectomy, J Cataract Refractive Surgery, Dec 1998, 24, pp. 1575-1579.

[8] Maguen, E., Machat, J.J., Complications of photorefractive keratectomy, primarily with the VISX excimer laser, Corneal Laser Surgery 1995, pp. 143-158. 